\title{
Neural Dynamics of Saccadic Suppression
}

\author{
Frank Bremmer, ${ }^{1,2}$ Michael Kubischik, ${ }^{1}$ Klaus-Peter Hoffmann, ${ }^{1}$ and Bart Krekelberg ${ }^{3}$ \\ ${ }^{1}$ Department of Zoology and Neurobiology, Ruhr-University Bochum, D-44780 Bochum, Germany, ${ }^{2}$ Department of Physics, Marburg University, D-35032 \\ Marburg, Germany, and ${ }^{3}$ Rutgers University, Center for Molecular and Behavioral Neuroscience, Newark, New Jersey 07102
}

We make fast, ballistic eye movements called saccades more often than our heart beats. Although every saccade causes a large movement of the image of the environment on our retina, we never perceive this motion. This aspect of perceptual stability is often referred to as saccadic suppression: a reduction of visual sensitivity around the time of saccades. Here, we investigated the neural basis of this perceptual phenomenon with extracellular recordings from awake, behaving monkeys in the middle temporal, medial superior temporal, ventral intraparietal, and lateral intraparietal areas. We found that, in each of these areas, the neural response to a visual stimulus changes around an eye movement. The perisaccadic response changes are qualitatively different in each of these areas, suggesting that they do not arise from a change in a common input area. Importantly, our data show that the suppression in the dorsal stream starts well before the eye movement. This clearly shows that the suppression is not just a consequence of the changes in visual input during the eye movement but rather must involve a process that actively modulates neural activity just before a saccade.

\section{Introduction}

While reading this article, your eyes jump from word to word. Each jump causes the retinal image to move. Normally, however, you are blissfully unaware of such motion: this is the behavioral phenomenon called saccadic suppression. Saccadic suppression is important to allow the perception of a stable environment (Helmholtz, 1867; Holt, 1903; Bridgeman et al., 1993; Ilg and Hoffmann, 1993). Psychophysical studies have demonstrated that not all visual stimuli are suppressed; stimuli processed by the functional subsystem that serves motion and space encoding are strongly suppressed, whereas stimuli processed by the object and color vision subsystem are essentially unimpacted (Ross et al., 1996). This behavioral dichotomy matches the two information processing streams in the primate visual system (Ungerleider and Mishkin, 1982).

Results from behavioral studies (Holt, 1903; Burr et al., 1994) and transcranial magnetic stimulation (Thilo et al., 2004) have been used to argue that the neural mechanism of saccadic suppression is a reduction of the input to the visual cortex at the level of the lateral geniculate nucleus (LGN). Physiological studies confirm that visually driven activity in the LGN is affected by eye movements. However, LGN neurons mainly show postsaccadic increases in response, and these effects are quite similar for the magnocellular and parvocellular subdivisions (Fischer et al., 1996; Reppas et al., 2002). It seems therefore that the perisaccadic changes in LGN response properties by themselves are not suffi-

Received June 18, 2009; revised Aug. 20, 2009; accepted Aug. 24, 2009.

This work was supported by Deutsche Forschungsgemeinschaft Grants SFB 509/B7 and F0R 560/A3, Human Frontiers Science Program Grant RG00149/1999-B, National Institutes of Health Grant R01EY017605, and European Union Research Grant MEMORY 6 FP/043236 NEST.

Correspondence should be addressed to Frank Bremmer, Department of Neurophysics, Marburg University, Renthof 7, D-35032 Marburg, Germany. E-mail: frank.bremmer@physik.uni-marburg.de.

DOI:10.1523/JNEUROSCI.2908-09.2009

Copyright $\odot 2009$ Society for Neuroscience ～0270-6474/09/2912374-10\$15.00/0 cient to explain the behavioral phenomenon of saccadic suppression and rather might be the consequence of top-down signaling from cortex to the thalamus. We therefore continued the search for neural correlates of saccadic suppression in the middle temporal (MT), medial superior temporal (MST), ventral intraparietal (VIP), and lateral intraparietal (LIP) areas of the macaque. We reasoned that, if saccadic suppression originates at the level of the LGN and cortical areas merely inherit this suppression, one would expect a relatively uniform effect of eye movements on the activity of neurons in all four areas. Our data do not support this view; instead, a unique pattern of saccadic suppression was found in each of these dorsal stream areas.

Not only is the locus of the neural basis of saccadic suppression uncertain, the mechanism of saccadic suppression is also poorly understood. Some have claimed that an active process reduces visibility even before the eyes start to move (Burr et al., 1994; Ross et al., 2001; Ibbotson et al., 2008), and others have concluded that the reduced visibility of presaccadic stimuli is caused mainly by backward masking of the visual scene that is present after the eye movement (Campbell and Wurtz, 1978; Ibbotson and Cloherty, 2009).

To shed light on this issue, we recorded the complete time course of neural excitability around saccadic eye movements. The time course we found in motion-sensitive areas MT, MST, and VIP was quite similar to what has been found psychophysically; neural suppression started for stimuli presented well before the saccade, reached a maximum at saccade onset, and, after a brief enhancement of neural responsiveness, returned to normal after the saccade. Neural suppression for stimuli that are presented to stationary eyes, just before they start to move, shows that active processes play a role in saccadic suppression.

These results have been published previously in abstract form (Bremmer et al., 2002), and a subset of the data has been used to construct a Bayesian model to decode position information from dorsal stream neurons (Krekelberg et al., 2003). 


\section{Materials and Methods}

The procedures for monkey training and electrophysiological recordings have been described in detail previously (Bremmer et al., 1997a,b; Schlack et al., 2005). In brief, monkeys were prepared for recordings under general anesthesia and under sterile surgical conditions. Each animal was implanted with a device for holding the head. A recording chamber for microelectrode penetrations through the intact dura was placed in a sagittal plane with an angle of $60^{\circ}$ with respect to the vertical for recordings in areas MT and MST and in a frontal plane at an angle of $45^{\circ}$ with respect to the vertical for recordings in areas LIP and VIP. Additionally, scleral search coils were implanted to monitor eye position. During the experiment, the animal sat in a primate chair with the head restrained, facing a translucent screen. All procedures were in accordance with published guidelines on the use of animals in research (European Communities Council Directive 86/609/EEC and National Institutes of Health Guide for the Care and Use of Laboratory Animals).

Visual stimulation. Visual stimuli and saccade targets were backprojected onto a screen subtending $60^{\circ} \times 60^{\circ}$ of the visual field. The animal received liquid rewards for correctly performing the saccade task. Saccades always had the same metric: along the horizontal meridian from $10^{\circ}$ left to $10^{\circ}$ right (in the second animal, from $10^{\circ}$ right to $10^{\circ}$ left). A brief visual stimulus (width, $10^{\circ}$; height, $60^{\circ}$; duration, $8 \mathrm{~ms}$ ) was presented at one of six neighboring but nonoverlapping locations (see Fig. 1). By stimulating the whole visual field across different trials, we could be sure that one of our stimuli always hit the most sensitive region within the receptive field. This avoids issues with shifting receptive fields that are known to occur in these areas (Duhamel et al., 1992, 1997; Nakamura and Colby, 2002). The flashed stimuli could appear long before (prestimulus), around (peristimulus), or long after (poststimulus) a saccade. Prestimuli occurred on average $550 \mathrm{~ms}$ before a saccade. Peristimulus occurrence ranged (in pseudorandomized order) from $200 \mathrm{~ms}$ before saccade onset to $200 \mathrm{~ms}$ after saccade onset. Poststimuli occurred on average $550 \mathrm{~ms}$ after a saccade.

In separate trials, the animal was instructed to make the same saccades, following the same visual cues, without the presentation of the vertical bar. Given the absence of strong visual stimulation, we refer to these trials as the saccade-only condition.

Data analysis. For the single-cell example in Figure 2, we aligned spike times to stimulus onset and sorted the trials by the time from stimulus onset to saccade onset. Spike density functions were generated by convolving the spike trains per trial with a Gaussian filter $(\sigma=5 \mathrm{~ms})$ and averaging across trials.

For the coarse-grained population analysis of Figure 3, we determined the response of each cell in a predefined response window (60-120 ms after stimulus onset). The average response in this window was considered the response of the cell. The average of the responses of all cells was then determined separately for all stimuli presented $>300 \mathrm{~ms}$ before (pre), or $>300 \mathrm{~ms}$ after (post) a saccade and for all stimuli presented from $100 \mathrm{~ms}$ before until $100 \mathrm{~ms}$ after saccade onset (peri). For ease of comparison across cells and areas, we expressed the average response of each cell in the peri- and post-periods as a percentage of the average response of that very cell in the pre-period.

For Figure 4, we calculated the time course of the average population discharge. Had we simply averaged the neural responses, then the variability in neural latencies (across cells and around eye movements) would have resulted in a smeared-out population response. Although this calculation would provide an accurate reflection of the activity in the brain, its ability to shed light on typical changes in response around saccades would be limited. Hence, for the computation of the population average, we corrected each cell for its response latency. To estimate the response latency of a cell, we first determined the mean and SD of the firing rate in the $200 \mathrm{~ms}$ before stimulus onset. Then, we determined the average response after stimulus onset in time bins of $20 \mathrm{~ms}$. The response latency was defined as the first bin in which the average response exceeded the baseline firing by at least 3 SDs. The smallest value across all stimulus positions was taken as the latency of the cell. This response latency was determined separately in the presaccadic, perisaccadic, and postsaccadic windows.
After correcting for the response latency of individual cells, we simply averaged the response across all cells in an area. Again, this was done separately for the presaccadic, perisaccadic, and postsaccadic window. The average stimulus presentation time in the pre-window was $550 \mathrm{~ms}$ before saccade onset, and in the post-window was $550 \mathrm{~ms}$ after saccade onset; for the peri-window, we chose the window of maximum suppression [the first $75 \mathrm{~ms}$ after saccade onset: $(0 \mathrm{~ms}, 75 \mathrm{~ms})$ ]. Confidence intervals (95\%) for the average of the population time course were set to 1.96 times the SEM of the population time course.

To calculate the population excitability of Figure 5, we aligned for each of the four areas all responses to saccade onset and averaged across all trials and neurons. We then cross-correlated the behavioral sensitivity data as reported by Diamond et al. (2000) with the neural activity profiles. This allowed us to determine the best match between behavioral and neural data. Neural response functions as obtained from motionsensitive areas MT, MST, and VIP were then corrected for the respective latencies and superimposed onto the behavioral data. Note that, for this analysis, we ignored when exactly a stimulus was presented. This provides an estimate of the average population activity at any point before, during, and after an eye movement. For each temporal epoch, this population activity estimate is based on the response to at least 750 flashed stimuli; hence, even if the number of flashes in each time window is not constant, our estimate of the average neural response will be precise.

For the cell-by-cell population analysis (see Fig. 6), we first grouped stimulus presentation times into three windows $(-1000 \mathrm{~ms},-400 \mathrm{~ms})$, $(-75 \mathrm{~ms}, 0 \mathrm{~ms})$, and $(0 \mathrm{~ms}, 75 \mathrm{~ms})$, in which the times were defined with respect to saccade onset. Accordingly, the first interval defines a presaccadic time window, whereas the second and third intervals define two different perisaccadic time windows. For each cell and each window, we then determined the maximum response for any of the flashed stimuli. With these values at hand, we calculated a suppression index (SI) (for additional details, see Results).

For a more fine-grained view of the time course, we determined the average population response separately for stimuli presented in $25 \mathrm{~ms}$ time windows around saccade onset (from $125 \mathrm{~ms}$ before until $175 \mathrm{~ms}$ after saccade onset). For each of these stimulus presentation windows, we determined the average activity from $280 \mathrm{~ms}$ before response onset until $300 \mathrm{~ms}$ after response onset. This results in a response matrix that is shown in Figure 7.

To quantify perisaccadic suppression, we compared the perisaccadic population response matrix of Figure 7 with the population responses evoked long before a saccade. We defined a response modulation index as the difference between the perisaccadic response and the presaccadic response, divided by the SE of the presaccadic response. To estimate statistical significance of this modulation index, we calculated how much stimuli presented in the $(-500 \mathrm{~ms},-300 \mathrm{~ms})$ window were suppressed compared with the stimuli presented in the $(-700 \mathrm{~ms},-500 \mathrm{~ms})$ window. Saccades should have no influence in this presaccadic window; hence, the suppression thus measured must be spurious and provides a null distribution against which to test the suppression in the perisaccadic window. We considered significant only those perisaccadic modulation (suppression) indices for which we could reject the null hypothesis that they could have been drawn from the presaccadic modulation index distribution. The threshold for significance was set to $p<0.01$, and, to correct for multiple comparisons, we selected only those regions of significance that consisted of a contiguous region of four or more significant time windows. Figure 8 shows the significant modulation indices: suppression (red) and enhancement (green). Note that, as a result of the correction for multiple comparisons, some time windows that appear very dark in Figure 7 nevertheless do not appear in the conservatively thresholded Figure 8.

LIP subpopulations. We distinguished among three subpopulations of LIP neurons by analyzing their response in the saccade-only condition. We first determined the average firing rate in a $200 \mathrm{~ms}$ time window centered on saccade onset (sacc) and compared this with the firing rate in a $100 \mathrm{~ms}$ time window long before the saccade, during steady fixation (fix). We then calculated a saccade modulation index as (sacc - fix)/ $($ sacc + fix $) \times 100 \%$. LIP neurons with a saccade modulation index above $10 \%$ were labeled as LIP positive $\left(\mathrm{LIP}^{+}\right)$; the response field of these 
neurons must have matched the direction of the saccade $(n=82)$. LIP neurons with a saccade modulation less than $-10 \%$ were classified as LIP $^{-}$; the response field of these neurons was presumably in the direction opposite to the saccade $(n=45)$. Neurons with a saccade modulation index between -10 and $+10 \%$ were classified as LIP- 0 . By definition, these had only minor purely saccade-driven modulations; they were mainly driven by visual input $(n=27)$.

\section{Results}

We recorded extracellularly from neurons in MT $(n=40), \operatorname{MST}(n=104), \operatorname{VIP}(n=$ $180)$, and LIP $(n=154)$ in four hemispheres of two macaque monkeys. In each trial, the monkey made a $20^{\circ}$ horizontal saccade, and a large vertical bar was flashed at one of six screen positions. These visual stimuli were presented in pseudorandomized order for $8 \mathrm{~ms}$ long before (pre), long after (post), or in the temporal vicinity of (peri) the saccade (Fig. 1).

\section{Saccadic suppression at the single-cell level}

In Figure 2, we illustrate the perisaccadic changes in neural responses with a recording from a single MT neuron. Flashes presented just to the right of the fovea either long before (Fig. 2A) or long after (Fig. 2B) a saccade lead to a robust response. The spike density (Fig. 2E) reaches peaks of $\sim 60$ spikes per second and is comparable for stimuli flashed in the presaccadic (red curve) and postsaccadic (blue curve) window. Figure $2 C$ shows the response of the same neuron to stimuli flashed in its presaccadic receptive field around the time of saccade onset. The trials in the raster plot are ordered such that trials in which the stimulus was presented long before the saccade are at the top, and trials in which the stimulus was presented long after the saccade are at the bottom. Saccade onset time is indicated by the red triangles.

Reading the raster from top to bottom, we see a robust response for flashes presented $100 \mathrm{~ms}$ or more before the saccade. Flashes presented between 75 and 0 ms before the saccade, however, evoke fewer spikes. The spike density for stimuli presented in this perisaccadic window is shown as the solid green curve in Figure $2 E$. The peak response does not rise above $35 \mathrm{~Hz}$, a reduction of nearly $50 \%$ compared with the response during fixation (red/blue curves). Because the eye has not moved yet when the flash is presented, this suppression of the response must involve an active process of suppression and cannot be caused by changes in the retinal input attributable to the motion of the eye.

Continuing stepping through the trials in Figure $2 C$, we reach the trials in which flashes are presented after the eye has started to move. The average response to these flashes (green dashed curve in Fig. 2E) is also much less than those presented during fixation. This reduction can be caused by active processes, but it almost certainly also involves the (passive) reduction in firing expected from the fact that the receptive field, which is presumed to be yoked to the eye, moves away from the position where the stimulus is flashed.

Figure $2 D$ shows the response to flashes presented in the future field, i.e., the location on the screen where the receptive field

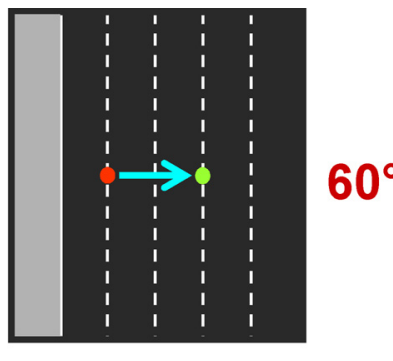

$60^{\circ}$

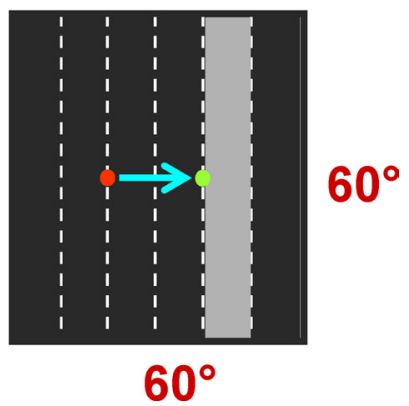

$60^{\circ}$

$60^{\circ}$

$60^{\circ}$ will be after the saccade. For flashes presented before the saccade, responses at this location are small and delayed (solid black curve in Fig. 2E). This likely includes a passive effect; when these flashes are presented, the receptive field does not yet encompass their location. It also could reflect a memory trace as described, e.g., in area LIP (Duhamel et al., 1992). However, the raster plot shows that, even for flashes presented $>50 \mathrm{~ms}$ after saccade onset, when the eye has reached its new position and the flashes are again presented within the receptive field, the response is also reduced compared with fixation (dashed black curve in Fig. 2E).

The single-cell example of Figure 2 demonstrates that it is critical to distinguish between the time of a response relative to the stimulus and the time of the stimulus relative to the saccade. Moreover, it illustrates how active sources of saccadic suppression can be isolated by investigating responses evoked by flashes presented just before or just after an eye movement. We believe, however, that passive sources of saccadic suppression may also play a role in perisaccadic perception. To illustrate this, consider an ideal observer with access to a perfect eye position signal. This observer, when faced with a detection task, always knows which neurons to consult about the presence of a visual stimulus on the screen. Therefore, this observer does not suffer from passive mechanisms of suppression because, as soon as the eye starts to move, the observer will select to read out a different neuron whose receptive field is now in the appropriate position. In contrast, any mechanism for detection that does not have access to a perfect eye position signal would suffer from passive suppression. For instance, if the eye position signal were delayed, the detection mechanism may retrieve information from a neuron whose receptive field no longer encompasses the stimulus (as in the bottom half of Fig. 2C). The passively reduced signal from this neuron could therefore increase the detection threshold of this mechanism. We believe that it is conceivable, even likely, that perceptual mechanisms have imperfect access to the current eye 
A

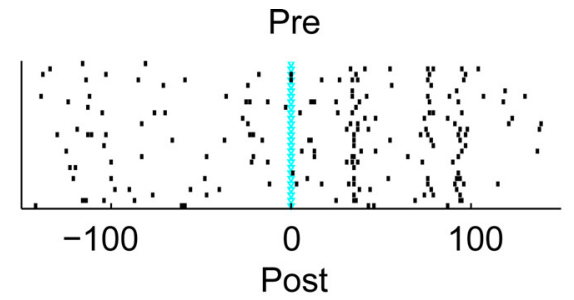

B

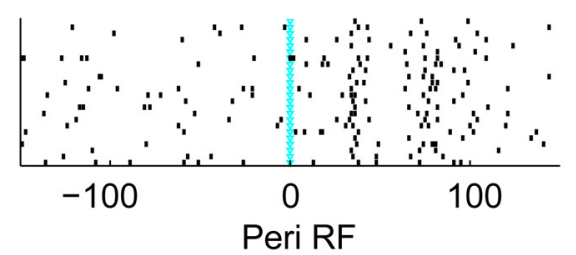

C

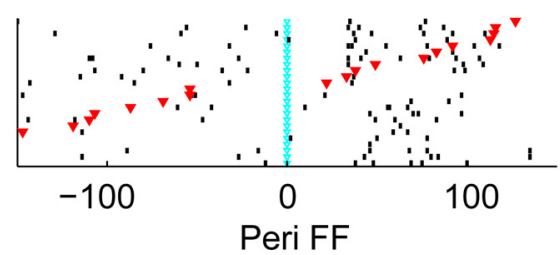

D
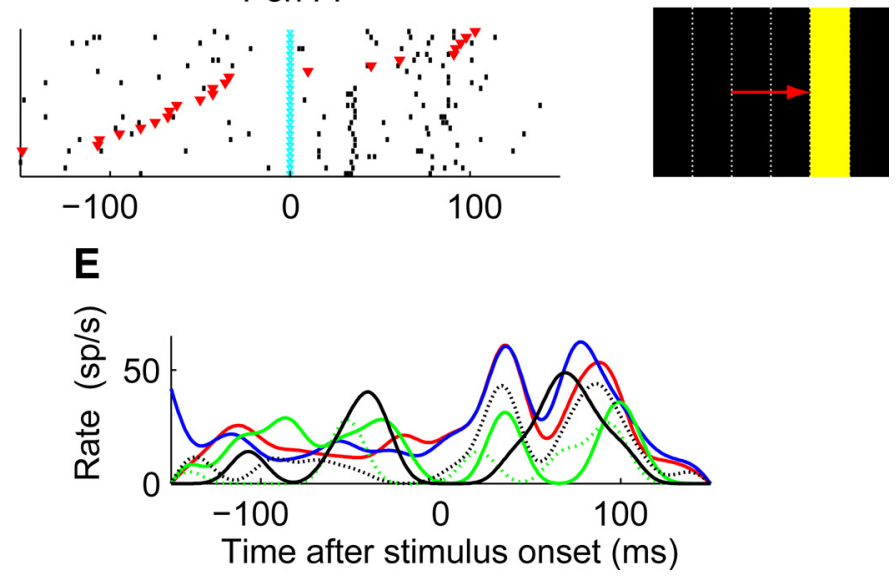

Figure 2. Saccadic suppression in an MT neuron. $A-D$, Response of a single neuron to a stimulus flashed at time 0; each black vertical tick corresponds to a single spike. The diagrams on the right show the screen (black rectangle), the position of the stimulus (yellow bar) on the screen, and the position of the eye (red dot for fixation, red arrow for saccades). $\boldsymbol{A}$, Response to stimuli flashed long before a saccade. The stimulus is flashed in the receptive field. $\boldsymbol{B}$, Response to stimuli flashed long after the $20^{\circ}$ rightward saccade. These flashes appear in the future field, i.e., the position of the receptive field after the saccade. $\boldsymbol{C}$, Response to stimuli flashed in the receptive field (RF), in the perisaccadic time window. The time of the saccade is indicated by the red triangles. For this negative (i.e., the flash came after the saccade had started) for the bottom-most trial. $\boldsymbol{D}$, Response to stimuli flashed in the perisaccadic window in the future field (FF). $\boldsymbol{E}$, Spike density calculated from $\boldsymbol{A}-\boldsymbol{D}$. The red (blue) curve represents the average response to pre (post)-saccadic stimuli. Green (black) curves represent the response to perisaccadic stimuli flashed in the receptive field as shown in $\boldsymbol{C}$ (future field; $\boldsymbol{D}$ ). Solid curves are average responses for flashes presented before the eye started to move [the difference between flash time and saccade onset was in the interval $(-75 \mathrm{~ms}, 0 \mathrm{~ms})]$. Dashed curves represent the average for flashes presented once the eye has started to move $(0 \mathrm{~ms}, 75 \mathrm{~ms})$. This neuron responded less to perisaccadic than to presaccadic or postsaccadic stimuli; the mechanisms involved in this are a mixture of active and passive processes (for details, see Results). representation, trials were sorted such that the time from stimulus flash to saccade onset was largest for the topmost trial and most

sponse separately for the four areas and for stimuli flashed long before (pre), near (peri), and long after (post) a saccade. The response in the pre-period was set to $100 \%$. Responsiveness in motionsensitive areas MT, MST, and VIP was less during saccades than during steady fixation. In areas MT and MST, perisaccadic activity was reduced to a level of $83 \%$ (area MT) and 88\% (area MST), whereas responses in area VIP were reduced to $91 \%$ of the presaccadic level. This difference was statistically significant in each area (repeated-measures ANOVA on ranks, followed by Tukey-Kramer post hoc tests, $p<0.05)$. Postsaccadic responses were enhanced to levels of 106\% (area MT), 106\% (area MST), and 108\% (area VIP). This effect reached statistical significance in both VIP and MST but not in MT.

In the LIP population as a whole, the average response did not differ significantly between the three periods (peri, 106\%; post, $104 \%$ ). Within this population of cells, however, some showed a strong increase in firing in the saccade-only condition (i.e., without any flashed visual stimuli), whereas others showed a strong decrease in the saccade-only condition. In other words, the average response shown in Figure $3 D$ is a mixture of pure saccade responses and visual responses. Given that almost all LIP cells in our population were modulated in the saccade-only condition, we are unable to perfectly isolate a pure visual response in the perisaccadic window. Within the subpopulation of cells in which the saccade alone evoked a response modulation of less than $\pm 10 \%$ (LIP-0; see Materials and Methods), however, the responsivity was qualitatively similar (peri, 108\%; post, 99\%).

Next, we calculated a time-resolved population response, by aligning the response of each neuron to response onset (i.e., we corrected for the individual latency of the cell). We ignored the spatial location of the flashed bars and simply averaged across all cells and trials; as such, this is an estimate of the overall population response. We determined this average separately for flashes presented in position and would therefore be expected to suffer from both passive and active suppression. For this reason, the following analysis first investigates the combined effect of passive and active suppression, in an attempt to show the overall reduction in sensitivity during eye movements (data shown in Figs. 3, 4). Subsequent analyses (Figs. 6-8) will study active suppression in isolation.

The joint effect of passive and active saccadic suppression At the coarsest level, we performed a population analysis that simply compared average activity per area in a fixed time window after stimulus onset. Figure 3 shows the average population re- three time windows: long before the saccade (pre), long after the saccade (post), and in the time window in which suppression was maximal (peri) ( $0 \mathrm{~ms}, 75 \mathrm{~ms}$ ). In this time window, both active and passive suppression mechanisms can operate. This analysis also allowed us to compare the visual response with the average response of these same neurons in the saccade-only condition (i.e., without any flashed bars). Figure 4 compares these presaccade, perisaccade, postsaccade, and saccade responses for the four populations of neurons. A comparison of the presaccadic and postsaccadic responses across areas shows that our visual stimuli led to clear and qualitatively comparable population responses in each area. Moreover, in each area, the presaccadic and postsac- 
cadic responses are approximately the same, which demonstrates the consistency of the responses, as well as the fact that the coverage of the population receptive field was approximately the same before and after the saccade.

In areas MT, MST, and VIP, the average perisaccadic responses were clearly reduced. The mechanisms underlying this suppression, however, are likely to be different in these areas. In areas MT and VIP, the neural response in the saccade-only condition was essentially unaffected by the saccade; hence, the suppression was specific to visual responses during the saccade. In the MST population, however, we found a clear reduction of activity even in the absence of visual stimuli (black curve). The suppression of visual activity during saccades (green curve) could at least qualitatively be explained as a linear summation of the suppression of activity evoked in the saccade-only condition (black curve) and the response to visual stimuli during fixation (red/blue curves).

The response pattern in LIP was quite different. Not unexpectedly, the saccade-only conditions led to large eye-movementrelated activity in LIP (black curve). Visual stimuli presented during the saccade could not drive the LIP population above this saccade-related activity and, at $\sim 50 \mathrm{~ms}$ after stimulus onset, even reduced the average LIP activity below the activity evoked in the saccade-only condition.

\section{Neural suppression matches \\ behavioral suppression}

The behavioral phenomenon of saccadic suppression has a well known time course. Our goal in this section is to determine whether changes in neural response in areas of the dorsal stream match this time course. To link neural activity with behavior, we determined what we call the population excitability (see Materials and Methods). This measure can be interpreted as the average neural response to a continuous stream of flashes presented to the retina. To allow a comparison across areas, we scaled the population excitability of each area at $\sim 100 \mathrm{~ms}$ before saccade onset to $100 \%$. Note that changes in this measure of population excitability around saccades include both active and passive mechanisms of suppression.

To relate the population excitability to the behavioral data obtained in humans, we made assumptions about the neural code that are necessarily tentative. We assumed that the neural response corresponds to the visibility of a stimulus that was presented some fixed amount of time earlier. With this assumption, the link between neural response and stimulus perception can be quantified by a single number: the latency. We estimated this latency by cross-correlating the behavioral sensitivity data with the neural activity profiles. We used the behavioral data of Diamond et al. (2000) and found that the temporal shift as obtained from this cross-correlation analysis was very similar but not identical for the three areas MT, MST, and VIP, respectively. In areas MT and MST, neurophysiological
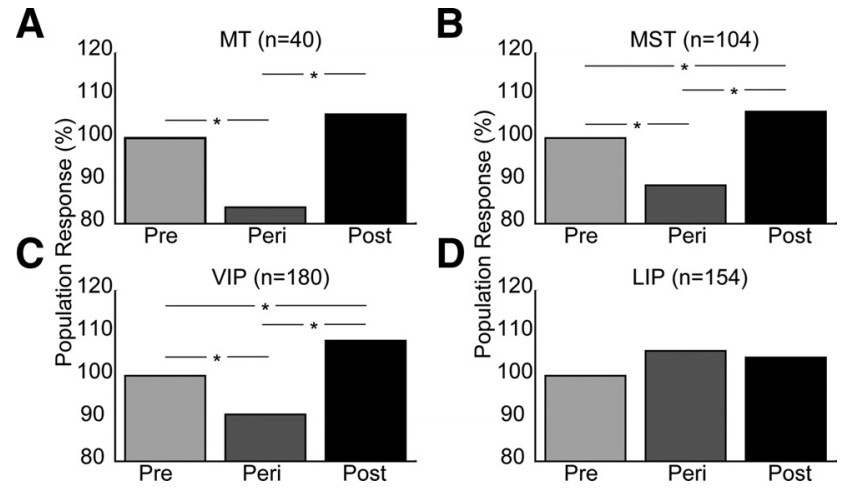

Figure 3. Comparison of visual responsiveness. In all four areas (A: MT; $\boldsymbol{B}$, MST; $\boldsymbol{C}, \mathrm{VIP} ; \boldsymbol{D}$, LIP), the median visual response during fixation long before a saccade was normalized to a value of $100 \%$. The response was computed for a preselected response window $(60-120 \mathrm{~ms}$ after stimulus onset). The horizontal lines and asterisks indicate significant differences $(p<0.05)$ as calculated by a repeated-measures ANOVA on ranks, followed by Tukey-Kramer-corrected post hoc tests (for details, see Results).

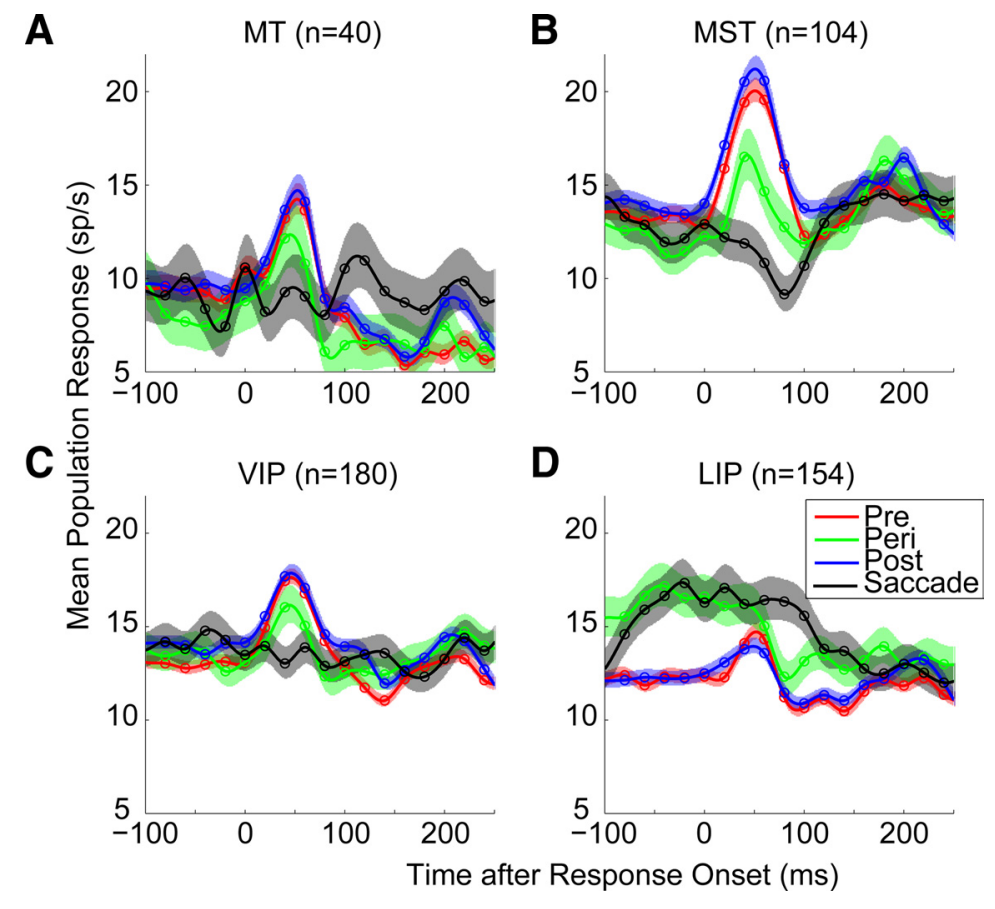

Figure 4. Time course of the stimulus response. Population average responses to bars flashed long before (red), during (green), or long after (blue) a saccade. These curves are aligned to the presentation of the flash, but for each cell, we corrected for its response latency. Accordingly, in each panel, a time value of zero indicates response onset. The black curve shows the average response of the same population to saccades made in darkness. For this curve, time 0 is saccade onset but is again corrected for the response latencies of individual cells to allow direct comparison with the visual responses of the other three curves. Circles show the data points calculated in independent time windows, curves show spline interpolation of those data points, and the shading around the curves indicates the $95 \%$ confidence intervals of the estimates of the mean population response. $\boldsymbol{A}$, MT population. $\boldsymbol{B}$, MST population. C, VIP population. D, LIP population. In LIP the visual stimulus reduced the response compared with the saccadeonly condition. In MT, MST, and VIP, the average perisaccadic response was reduced compared with the presaccadic and postsaccadic responses. sp/s, Spikes per second. data had to be shifted by $\tau=98 \mathrm{~ms}$ to obtain an optimal match with the behavioral data [maximum of the normalized crosscorrelation coefficient: $r_{\mathrm{Max}}=0.6153$ (area MST) and $r_{\mathrm{Max}}=$ 0.6073 (area MT)]. Data from area VIP had to be shifted by $\tau=112$ $\mathrm{ms}\left(r_{\mathrm{Max}}=0.6087\right)$. In other words, if perception were based on neural activity $\sim 100 \mathrm{~ms}$ after stimulus onset, then the suppression of neural activity in areas MT, MST, and VIP would be a good predictor of perisaccadic reductions of visibility. In 


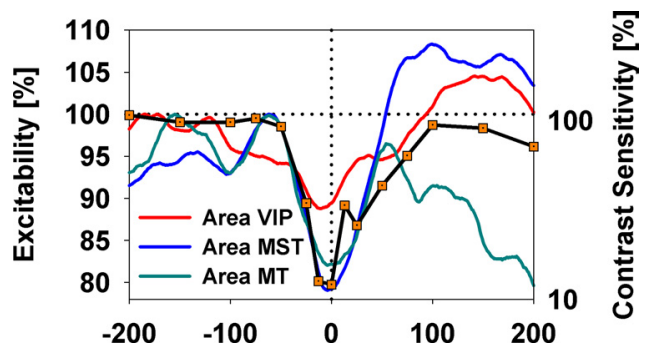

Figure 5. Comparison of neural and behavioral data. For the behavioral data, the horizontal axis shows time relative to saccade onset, and the right vertical axis indicates normalized contrast sensitivity as taken from the study by Diamond et al. (2000). Neuronal data were shifted along the time axis to correct for response and processing latencies (see Results) and represent neuronal excitability (left vertical axis) of the MT and MST populations (cyan and blue curves, respectively) and the VIP population (red curve). The time course of neuronal excitability in all three motion areas of the macaque shows a good qualitative match with the time course of perceptual loss of sensitivity around saccades in human subjects.
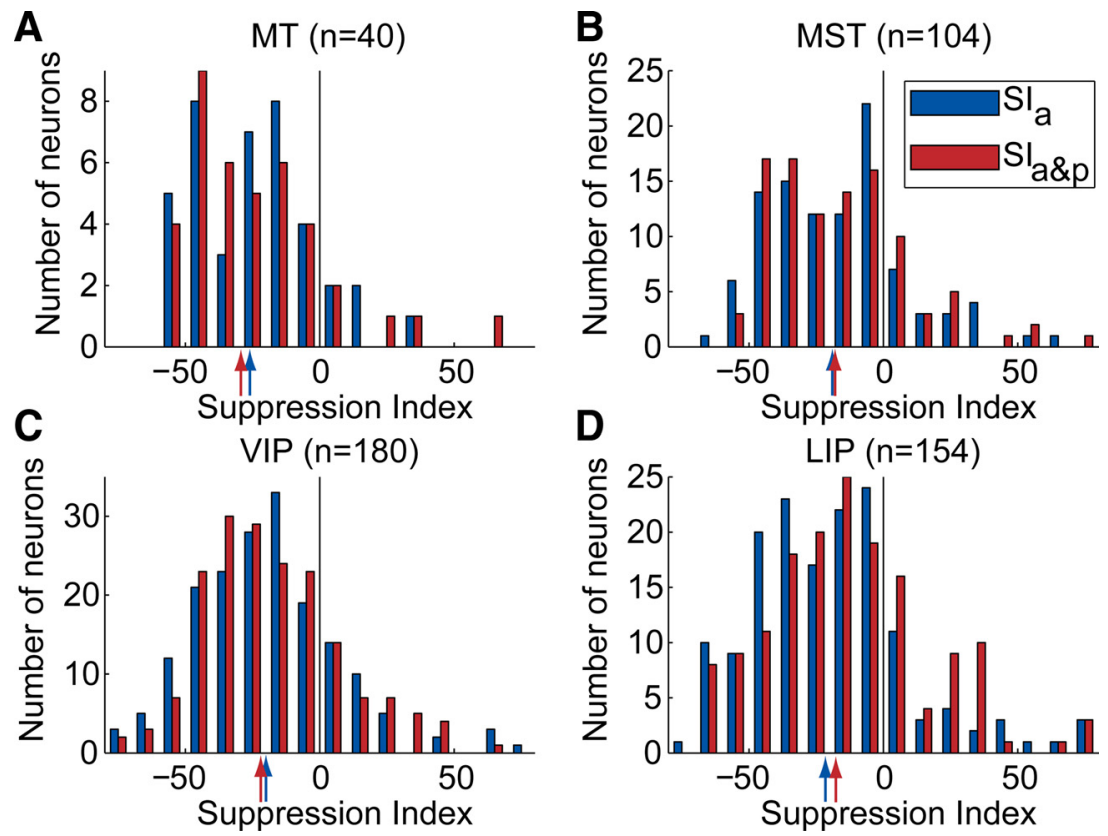

Figure 6. Comparison of perisaccadic and presaccadic peak responses. We compared the maximum response that could be evoked by a stimulus (at any position on the screen) long before a saccade $(-1000,-400 \mathrm{~ms})$ with the maximum response to stimuli presented just before $(-75,0 \mathrm{~ms})$ or during $(0,75 \mathrm{~ms})$ a saccade. For both perisaccadic intervals, we computed for each cell a suppression index (for definition, see Results). $\boldsymbol{A}-\boldsymbol{D}$ show the distribution of the suppression indices for the two temporal intervals. $\boldsymbol{A}$, The population of MT neurons. $\boldsymbol{B}$, The population of MST neurons. $\boldsymbol{C}$, The population of VIP neurons. $\boldsymbol{D}$, The population of LIP neurons. The population medians are indicated by the blue (just before) and red (during) arrows in each panel. In all four populations, the median suppression index was significantly below zero $(p<0.001)$ for stimuli presented just before and during saccades. In all but the LIP population, the median peak responses for stimuli shown briefly before and during the saccade were not significantly different $(p>0.6)$. Saccadic suppression in the dorsal stream therefore starts for stimuli presented before the eyes start to move and does not significantly change during the eye movement.

Figure 5, we shifted the ongoing population activity of areas MT and MST by $\tau=98 \mathrm{~ms}$ and population activity of area VIP by $\tau=112 \mathrm{~ms}$ and overlaid it with the behavioral sensitivity data of Diamond et al. (2000).

\section{Temporal dynamics of saccadic suppression}

Psychophysical studies have investigated the temporal evolution of saccadic suppression by measuring contrast sensitivity for luminance gratings presented shortly before, during, or after saccades (Diamond et al., 2000). The suppression of contrast sensitivity starts for stimuli presented well before saccade onset and is maximal for stimuli presented at the time the eyes start to move. Contrast sensitivity returns back to control level for stimuli presented $\sim 50-100 \mathrm{~ms}$ after saccade offset. Although this shows that presaccadic stimuli are suppressed, this does not by imply that the saccadic suppression mechanism must hence, there is ample time for intra-saccadic and postsaccadic echanisms to change the behavioral response to the presaccadic stimulus. Behavioral methods or imaging methods relysuffer from this delay between the measurement and the stimulus presentation time. In an electrophysiological recording, this delay is uced to the response latency of the cell (plus minimal latencies in the recording equipment). This allowed us to address the temporal dynamics of suppression with high fidelity.

In this section, we focus on the perisaccadic time window. First, we quantify suppression on a cell-by-cell basis (Fig. 6), and then we show the average population response for stimuli flashed at different times with respect to saccade onset (see Figs. 7, 8).

\section{Active saccadic suppression}

The response of the example cell in Figure 2 was reduced even for flashes that were presented to a stationary retina. This is what we refer to as active suppression. To quantify the strength of active and passive suppression across areas, we determined the peak of the response for stimuli flashed long before a saccade and compared that with the peak response evoked by stimuli flashed (1) just before or (2) during a saccade. Determining the peak response across spatial positions of the flashed bar ensured that we always compared stimuli flashed in the most sensitive part of the receptive field, regardless of when and where the receptive field moved relative to the saccade. We calculated a suppression index as follows: $\mathrm{SI}=$ [(perisaccadic response/presaccadic response) -1 ] $\times 100 \%$. An SI of zero corresponds to a cell whose peak firing rate is not affected by the saccade; a negative SI corresponds to saccadic suppression, whereas a positive SI corresponds to saccadic enhancement. We calculated these indices separately for the two perisaccadic time windows. The effects in the first perisaccadic time window $(-75 \mathrm{~ms}, 0 \mathrm{~ms})$ are attributable to pure active suppression; we refer to them as $\mathrm{SI}_{\mathrm{a}}$. The effects in the second perisaccadic time window ( $0 \mathrm{~ms}, 75 \mathrm{~ms})$ include both active and passive mechanisms; we refer to them as $\mathrm{SI}_{\mathrm{a} \& \mathrm{p}}$.

Figure $6 A-D$ shows histograms of the suppression indices for each of the populations. Overall, the magnitude of saccadic suppression was similar in range across areas, ranging from $\sim 60 \%$ suppression to $\sim 40 \%$ enhancement. The median indices [indicated by blue (before, $\mathrm{SI}_{\mathrm{a}}$ ) and red (during, $\mathrm{SI}_{\mathrm{a} \& \mathrm{p}}$ ) arrows in each panel] were always below zero; hence, perisaccadic peak re- 
sponses were typically reduced compared with presaccadic peak responses. The median suppression index for stimuli presented just before the saccade $\left(\mathrm{SI}_{\mathrm{a}}\right)$ was as follows: MT, $-26 \%$; MST, $-19 \%$; VIP, $-20 \%$. The median suppression for combined active and passive mechanisms $\left(\mathrm{SI}_{\mathrm{a \& p}}\right)$ was as follows: MT, $-29 \%$; MST, $-18 \%$; VIP, $-22 \%$. These reductions in peak response compared with the presaccadic peak responses were statistically significant in both perisaccadic time windows and in all areas $(p<0.001$, paired sign test). The difference in reduction between the two time windows was not significant in MT, MST, or VIP ( $p>$ 0.6 , paired sign test).

In LIP, both active and combined active and passive suppression indices were also significantly below zero $\left(\mathrm{SI}_{\mathrm{a}}\right.$ of $-22 \%$; $\mathrm{SI}_{\text {a\&p }}$ of $-18 \% ; p<0.001$, paired sign test). Note that these data may appear to contradict the findings shown in Figure 4 in which we showed that the average perisaccadic response in LIP (green curve) is higher than the average presaccadic response (red curve). Here we show that the peak perisaccadic response is lower than the peak presaccadic response. The resolution of this paradox lies in the fact that the LIP response in the perisaccadic windows most likely is a motor response that is independent of the position of the flash; hence, its average over all positions (shown in Fig. 4) is higher than the average taken long before the eye movement when the response is truly visual and restricted to a few locations on the screen. Therefore, a different way of phrasing the LIP results is that visual stimuli drive the cells during fixation but that the same visual stimuli cannot increase the response during or just before a saccade, when the cell is already responding strongly in response to the saccade itself. In fact, if anything, the visual stimuli reduce the saccade response.

Consistent with this, we found that the suppression index during the saccade was slightly less negative than just before the saccade $\left(\mathrm{SI}_{\mathrm{a \& p}}>\mathrm{SI}_{\mathrm{a}} ; p<0.01\right.$, paired sign test). This is presumably attributable to the fact that most of our LIP cells increased their firing perisaccadically even in the saccade-only condition. To confirm this, we performed this analysis separately for the subpopulation of LIP cells that had enhanced responses in the saccade-only condition $\left(\mathrm{LIP}^{+}\right)$, those that reduced their responses during saccades $\left(\mathrm{LIP}^{-}\right)$, and those that were only modestly modulated by saccades (LIP-0) (see Materials and Methods). In the $\mathrm{LIP}^{+}$population, the suppression index was $-15 \%(p<0.01)$ for both time windows. In the $\mathrm{LIP}^{-}$population, $\mathrm{SI}_{\mathrm{a}}$ was increased to $-43 \%(p<0.01)$, whereas $\mathrm{SI}_{\mathrm{a} \& \mathrm{p}}$ was $-31 \%(p<0.01)$. In the LIP-0 population, $\mathrm{SI}_{\mathrm{a}}$ was $-23 \%(p<$ $0.01)$ and $\mathrm{SI}_{\mathrm{a \& p}}$ was $-10 \%(p>0.05)$. This shows that, in the perisaccadic time window, the LIP population as a whole but also each of its subpopulations is driven mainly by saccade planning; visual stimuli that arrive around this time are actively suppressed.

To provide additional insight into the detailed temporal dynamics of the population activity around a saccade, Figure 7 displays the full time courses as two-dimensional activity maps. The two dimensions correspond to two important temporal factors that determine the neural response: the time of stimulus presentation relative to saccade onset (horizontal) and the time relative to response onset (vertical). Zero on the horizontal axis corresponds to stimuli that land on the retina when the saccade starts. Positive values indicate that the stimulus was presented after saccade onset. Zero on the vertical axis is the time at which the first response can reliably be detected in a cell (i.e., the response time is corrected for the latency of each cell).

There are two important features to note in this diagram. First, the horizontal yellow-red bands in Figure $7 A-C$ show that, in areas MT, MST, and VIP, the stimulus triggered a clear response that lasted $\sim 70 \mathrm{~ms}$. This response, however, was reduced for stimuli presented just before and during the saccade. This reinforces the finding described in Figure 4 and shows that a reduction in visual activity is found for stimuli presented around saccade onset.

The second feature of interest is most notable in MST. A diagonal dark band of suppressed activity aligns with the black line indicating saccade onset. Given that this suppression starts before saccade onset, it cannot be caused by changes in the retinal stimulation but must be attributable to an active process of suppression. Neurons in area LIP show a quite different response pattern: the population shows strong saccade-related activity (yellow-red diagonal band) but responds little if at all to the stimulus (absence of a horizontal band). This occurs although this same population responds quite well to visual stimuli during fixation (not shown in this format, but see Fig. 4).

We performed a statistical analysis (see Materials and Methods) to determine when the perisaccadic neural response (Fig. 7) was different from the response to visual stimuli long before a 
A
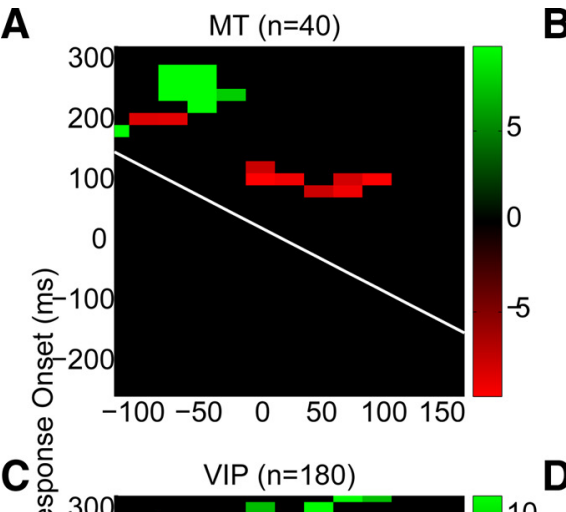

B

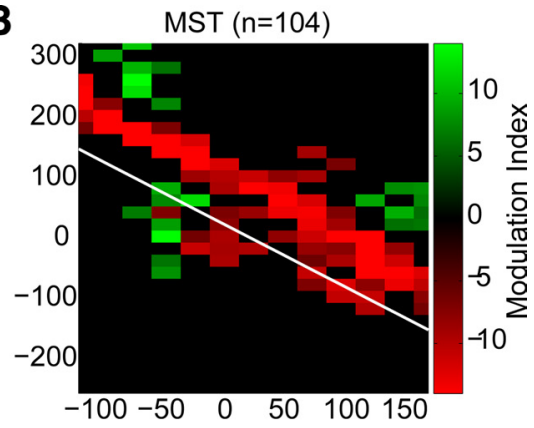

D

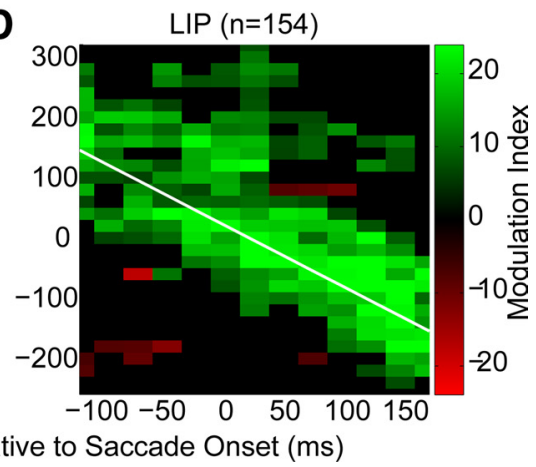

Figure 8. Suppression map. The horizontal axis represents the time from stimulus onset relative to saccade onset, and the vertical axis represents the time relative to response onset. The color represents a statistical estimate of the suppression/enhancement measured relative to stimuli presented long before a saccade (see Materials and Methods). The diagonal white line shows the time at which the saccade started. $\boldsymbol{A}$, Suppression and enhancement in the MT population. $\boldsymbol{B}$, Suppression and enhancement in the MST population. C, Suppression and enhancement in the VIP population. D, Suppression and enhancement in the LIP population.

saccade. Figure 8 shows the results of this analysis in approximately the same format as Figure 7; the main difference is that, in this representation, red shows a statistically significant suppression, and green indicates a significant enhancement of perisaccadic activity (see Materials and Methods). This statistical thresholding reveals a much clearer pattern of suppression. To refer to specific parts of these maps, we use $t_{x}$ to refer to the time of stimulus presentation relative to saccade onset ( $x$-axis) and $t_{y}$ to the time after response onset ( $y$-axis).

In area MT, the main features are a saccadic enhancement (green) of stimuli presented 100 to $50 \mathrm{~ms}$ before the saccade $\left[t_{x}=\right.$ $\left.(-100 \mathrm{~ms},-50 \mathrm{~ms}) ; t_{y}=\sim 250 \mathrm{~ms}\right]$ and a suppression (red) of the response to stimuli presented perisaccadically $\left[t_{x}=(-20 \mathrm{~ms}, 100\right.$ $\mathrm{ms}) ; t_{y}=\sim 80 \mathrm{~ms}$.

In area MST, we see an enhancement (green) of the response to stimuli presented before the saccade $\left[t_{x}=(-100 \mathrm{~ms}, 0 \mathrm{~ms})\right.$; both near $t_{y}=\sim 0$ and $\left.\sim 250 \mathrm{~ms}\right]$, as well as an enhancement of responses for stimuli presented after the saccade $\left(t_{x}=>100 \mathrm{~ms}\right.$; $\left.t_{y}=\sim 50 \mathrm{~ms}\right)$. The main feature, however, is a trough of suppression (red) that starts before saccade onset and extends well beyond the saccade. The alignment with the diagonal white line of saccade onset and the presence of significant suppression before stimulus response onset $\left(t_{y}=0 \mathrm{~ms}\right)$ shows that this suppression is saccade related and an active process that takes place even without the presence of visual responses. Additionally, the fact that this suppression is significant even before the onset of the saccade (white line) shows that this suppression cannot be attributable to the retinal slip caused by the saccades.

In area VIP, all significant suppression (red) was observed after stimulus response onset and never in the absence of visual stimuli. The strongest enhancement (green) was found in the late $\left(t_{y}=\sim 250 \mathrm{~ms}\right.$ ) response to stimuli presented $100 \mathrm{~ms}$ before until
$50 \mathrm{~ms}$ after saccade onset $\left[t_{x}=(-100 \mathrm{~ms}\right.$, $50 \mathrm{~ms})$ ]. A weaker enhancement appears to precede saccade onset, even before the visual response.

In area LIP, the statistical analysis confirms that cells are typically more strongly driven by (impending) saccades than by visual stimuli. The isolated island of suppression near $t_{x}=50 \mathrm{~ms}, t_{y}=50 \mathrm{~ms}$ corresponds to the reduction in response after a visual stimulus presented during a saccade visible in Figure 4.

\section{Discussion}

We investigated neural correlates of saccadic suppression in the dorsal stream of the macaque visual cortical system. In each of the investigated areas, we found clear interactions between the visual response and the eye movements that reflect suppression of neural activity. First, the pattern of suppression was qualitatively different in each of the areas; this is at odds with an early cutoff of visual input to the cortex during saccades. Second, we found that reductions in firing rate can be observed well before the eye starts to move and even independently of the presence of a visual stimulus. This shows that at least part of the neural suppression must be attributable to an active process independent of intra-saccadic or postsaccadic changes in the visual input caused by the saccade. Third, we showed that the time course of neural excitability in the motionsensitive areas of the dorsal stream matched well with the time course of perceptual suppression in humans. This strengthens the case that these neural changes are indeed neural correlates of the behavioral phenomenon of saccadic suppression. We will discuss these findings in light of previous behavioral, imaging, and physiological studies of saccadic suppression.

Much of the psychophysical debate has centered on the question whether saccadic suppression is an active process or not (Castet et al., 2001; Ross et al., 2001; Vallines and Greenlee, 2006). In other words, does the visual system actively attenuate processing, or is the reduced visibility a consequence of the changes in the retinal input caused by the eye movements themselves? The fact that presaccadic stimuli are perceptually suppressed does not necessarily imply that suppression is an active process. The reason for this is that the subject's response always occurs well after the eye movement and can therefore be influenced by intrasaccadic retinal image motion or the visual input evoked by the landing of the eye at the new position (Campbell and Wurtz, 1978). Behavioral studies clearly show that such passive, retinal processes play an important role in saccadic suppression (Campbell and Wurtz, 1978). Previous physiological studies have shown presaccadic changes in receptive field size and location; hence, there can be no doubt that active processes play a role in visual processing around eye movements (for review, see Ross et al., 2001; Wurtz, 2008). The more specific question, whether an active process underlies saccadic suppression, however, is more difficult to answer. Our approach was to use the same visual stimulus to map the full temporal dynamics of changes in responsivity around saccades in four dorsal stream areas. This allowed us to show that a presac- 
cadic, active, extraretinal suppression process operates in the areas strongly associated with motion perception.

Moreover, our approach of using the same stimulus in multiple areas provided us with a measure of neural suppression that could be compared across areas. Because the qualitative effects were similar across motion areas, we believe that it is reasonable to expect that these neural changes would have significant perceptual effects. In Figure 5, we compared the neural data obtained from motion areas with behavioral data obtained in humans and found a striking resemblance. Although the comparison was across species, we believe that this strengthens the claim that these neural changes may indeed be responsible for the behavioral changes associated with saccadic suppression. Nevertheless, future work that performs a direct trial-by-trial comparison of neural activity and the perceptual report of the animal is needed to demonstrate a causal link between the neural and behavioral phenomena of saccadic suppression.

Previous physiological studies in macaque area MT have reported a number of perisaccadic changes in responsivity. Thiele et al. (2002) reported that some MT cells reduced their firing rate during eye movements. Consistent with this, a reduced BOLD response to luminance modulated stimuli has also been reported in human MT (Kleiser et al., 2004). Our findings confirm a general reduction in the response of MT and MST cells around saccades but, additionally and most importantly, allow us to demonstrate that these changes start before the saccade.

In agreement with previous findings concerning the dorsal aspect of area MST (Ibbotson et al., 2007, 2008), we find a postsaccadic enhancement of the neural response in areas MT and MST. Additionally, we show that such an enhancement also occurs in VIP. There are several interesting hypotheses about the functional role of this enhancement. First, as Ibbotson et al. (2007) have suggested, this enhancement could be related to the enhanced postsaccadic ocular following response reported by Kawano and Miles (1986). In monkeys, area MST is involved in the execution of these short-latency eye movement responses (Inoue et al., 1998). Moreover, these areas are known to be involved in motion perception (for review, see Born and Bradley, 2005; Britten, 2008); hence, one could expect an enhanced neural response to result in an enhanced perceptual sensitivity. There is some evidence in the behavioral literature to support this view (Diamond et al., 2000, their Fig. 4), but this phenomenon has not yet been studied in detail. Finally, Ibbotson and Cloherty (2009) have recently suggested that postsaccadic increases in firing could be the neural source of backward masking. In this view, the enhanced response to the postsaccadic scene helps to remove the weak response to perisaccadic stimuli from awareness.

An important difference between our findings in MST and those of Ibbotson et al. (2008) is that we find suppression when the animal makes saccades in darkness, whereas Ibbotson et al. find no evidence for this. The most likely reason for this discrepancy is that "darkness" in our case was not as complete as that of Ibbotson et al. In our experimental setup, the saccades in darkness were made in response to a small red dot, and the video projector (projecting "black") was on; hence, the projection screen was not completely dark. We note, however, that because suppression in MST starts before saccade onset (Fig. 8), the suppression cannot be attributable to saccade-induced retinal slip. Together, the two studies suggest that an active process of suppression takes place in MST even before the saccade starts but only if there is at least some residual visual input.

We are not aware of any previous studies that have addressed these issues in VIP. Our data show that VIP responds in a manner that is quite similar to MT and MST. The main difference appears to be that both suppression and postsaccadic enhancement in VIP occur later. A more mechanistic difference is that, in MST, the response to visual stimuli during a saccade is well described by the linear sum of the activity in the saccade-only condition and the response to visual stimuli during fixation. Conversely, in VIP and MT, this interaction must be distinctly nonlinear because there was no significant suppression of activity in the saccadeonly conditions. We see this as additional evidence that partially separate mechanisms must underlie the perisaccadic changes in visual responses in these areas.

The high perisaccadic activity in LIP should not be misconstrued as evidence for the absence of suppression. On the contrary, with our visual stimuli, it was not possible to drive LIP cells beyond the activity they had during saccades in darkness. In other words, suppression of the visual response in LIP was 100\%. For the later response windows, the presence of a visual stimulus even led to a reduction of the neural response below the level seen during saccades in darkness. Activity in LIP, however, is not typically associated with direct visual perception of stimuli but rather with the allocation of attention (Colby and Goldberg, 1999; Bisley and Goldberg, 2003). If LIP activity reflects a mechanism that allocates spatial attention, the fact that these cells cannot be driven by visual stimuli at that time may be related to the behavioral phenomenon of the attentional blink. In this interpretation, LIP does not process attentional cues while attention is being reallocated (i.e., LIP is already active). Such a mechanism could be useful to prevent the saccade-generated retinal motion from being interpreted as an exogenous cue for attention.

This view further highlights the fact that different areas, with different functional roles, may have different demands on perisaccadic signals. Some areas may require a complete cutoff of input, whereas others may still be able to extract useful information from the incoming retinal signal. It is conceivable, for instance, that the retinal motion signal generated by the saccade is used as a source of information about the speed and direction of the saccade. Rather than a nuisance, that information could be useful for the mechanism that constructs perceptual stability, as long as the motion signals are sufficiently dampened to prevent them from reaching awareness (Watson and Krekelberg, 2009). This view of perceptual stability and perisaccadic processing is consistent with the idea that the behavioral reduction of visibility around saccades is not attributable to an early (thalamic) cutoff of all visual input to the cortex. Instead of such an early cutoff, our new data suggest that there can be multiple separate mechanisms that adjust the perisaccadic visual signals to match the requirements of processing in a particular area.

\section{References}

Bisley JW, Goldberg ME (2003) Neuronal activity in the lateral intraparietal area and spatial attention. Science 299:81-86.

Born RT, Bradley DC (2005) Structure and function of visual area MT. Annu Rev Neurosci 28:157-189.

Bremmer F, Ilg UJ, Thiele A, Distler C, Hoffmann KP (1997a) Eye position effects in monkey cortex. I. Visual and pursuit-related activity in extrastriate areas MT and MST. J Neurophysiol 77:944-961.

Bremmer F, Distler C, Hoffmann KP (1997b) Eye position effects in monkey cortex. II. Pursuit- and fixation-related activity in posterior parietal areas LIP and 7A. J Neurophysiol 77:962-977.

Bremmer F, Kubischik M, Hoffmann KP, Krekelberg B (2002) Neural dynamics of saccadic suppression. Soc Neurosci Abstr 28:57.2.

Bridgeman B, van der Heijden AH, Velichkovsky BM (1994) Visual stability and saccadic eye movements. Behav Brain Sci 17:247-258.

Britten KH (2008) Mechanisms of self-motion perception. Annu Rev Neurosci 31:389-410. 
Burr DC, Morrone MC, Ross J (1994) Selective suppression of the magnocellular visual pathway during saccadic eye movements. Nature 371: 511-513.

Campbell FW, Wurtz RH (1978) Saccadic omission: why we do not see a grey-out during a saccadic eye movement. Vision Res 18:1297-1303.

Castet E, Jeanjean S, Masson GS (2001) "Saccadic suppression": no need for an active extra-retinal mechanism. Trends Neurosci 24:316-318.

Colby CL, Goldberg ME (1999) Space and attention in parietal cortex. Annu Rev Neurosci 22:319-349.

Diamond MR, Ross J, Morrone MC (2000) Extraretinal control of saccadic suppression. J Neurosci 20:3449-3455.

Duhamel JR, Colby CL, Goldberg ME (1992) The updating of the representation of visual space in parietal cortex by intended eye movements. Science 255:90-92.

Duhamel JR, Bremmer F, BenHamed S, GrafW (1997) Spatial invariance of visual receptive fields in parietal cortex neurons. Nature 389:845-848.

Fischer WH, Schmidt M, Stuphorn V, Hoffmann KP (1996) Response properties of relay cells in the A-laminae of the cat's dorsal lateral geniculate nucleus after saccades. Exp Brain Res 110:435-445.

Helmholtz H (1867) Handbuch der physiologischen Optik. Leipzig, Germany: Voss.

Holt E (1903) Eye movement and central anesthesia. Harvard Psychological Studies 1:3-45.

Ibbotson MR, Cloherty SL (2009) Visual perception: saccadic omissionsuppression or temporal masking? Curr Biol 19:R493-R496.

Ibbotson MR, Price NS, Crowder NA, Ono S, Mustari MJ (2007) Enhanced motion sensitivity follows saccadic suppression in the superior temporal sulcus of the macaque cortex. Cereb Cortex 17:1129-1138.

Ibbotson MR, Crowder NA, Cloherty SL, Price NS, Mustari MJ (2008) Saccadic modulation of neural responses: possible roles in saccadic suppression, enhancement, and time compression. J Neurosci 28:10952-10960.

Ilg UJ, Hoffmann KP (1993) Motion perception during saccades. Vision Res 33:211-220.

Inoue Y, Takemura A, Kawano K, Kitama T, Miles FA (1998) Dependence of short-latency ocular following and associated activity in the medial superior temporal area (MST) on ocular vergence. Exp Brain Res 121:135-144.

Kawano K, Miles FA (1986) Short-latency ocular following responses of monkey. II. Dependence on a prior saccadic eye movement. J Neurophysiol 56:1355-1380.

Kleiser R, Seitz RJ, Krekelberg B (2004) Neural correlates of saccadic suppression in humans. Curr Biol 14:386-390.

Krekelberg B, Kubischik M, Hoffmann KP, Bremmer F (2003) Neural correlates of visual localization and perisaccadic mislocalization. Neuron 37:537-545.

Nakamura K, Colby CL (2002) Updating of the visual representation in monkey striate and extrastriate cortex during saccades. Proc Natl Acad Sci U S A 99:4026-4031.

Reppas JB, Usrey WM, Reid RC (2002) Saccadic eye movements modulate visual responses in the lateral geniculate nucleus. Neuron 35:961-974.

Ross J, Burr D, Morrone C (1996) Suppression of the magnocellular pathway during saccades. Behav Brain Res 80:1-8.

Ross J, Morrone MC, Goldberg ME, Burr DC (2001) Changes in visual perception at the time of saccades. Trends Neurosci 24:113-121.

Schlack A, Sterbing-D’Angelo SJ, Hartung K, Hoffmann KP, Bremmer F (2005) Multisensory space representations in the macaque ventral intraparietal area. J Neurosci 25:4616-4625.

Thiele A, Henning P, Kubischik M, Hoffmann KP (2002) Neural mechanisms of saccadic suppression. Science 295:2460-2462.

Thilo KV, Santoro L, Walsh V, Blakemore C (2004) The site of saccadic suppression. Nat Neurosci 7:13-14.

Ungerleider LG, Mishkin M (1982) Two cortical visual systems. In: Analysis of visual behavior (Ingle DJ, Goodale MA, Mansfield RJW, eds), pp 549586. Cambridge, MA: MIT.

Vallines I, Greenlee MW (2006) Saccadic suppression of retinotopically localized blood oxygen level-dependent responses in human primary visual area V1. J Neurosci 26:5965-5969.

Watson TL, Krekelberg B (2009) The relationship between saccadic suppression and perceptual stability. Curr Biol 19:1040-1043.

Wurtz RH (2008) Neuronal mechanisms of visual stability. Vision Res 48: 2070-2089. 